\title{
Current Challenges Faced by Cancer Clinical Trials in Addressing the Problem of Under-Representation of Older Adults: A Narrative Review
}

\author{
Ruth M. Parks · Holly M. Holmes · Kwok-Leung Cheung
}

Received: November 19, 2020 / Accepted: January 9, 2021 / Published online: January 22, 2021

(C) The Author(s) 2021

\section{ABSTRACT}

The number of older adults living with cancer is increasing. There is a clear lack of representation of older adults in clinical trials, including cancer trials. Reasons for this are multifactorial and complex and include protocol, patient and sponsor factors. Potential solutions to overcome issues with trial design include varied methods of recruitment with flexible inclusion criteria. Possible alternatives to randomised trials include prospective cohort studies, pragmatic trials and the use of national population-based data sets. Patient factors may be addressed by integration of geriatric assessment, so patients can be randomised or treated based on their individual needs. Additionally, standard protocols for including older adults with cognitive impairment should be developed, rather than automatic exclusion. Increased effort is needed from sponsors and governing health care bodies to make recruitment of older adults to clinical trials standard.

R. M. Parks · K.-L. Cheung ( $₫)$

Nottingham Breast Cancer Research Centre, School

of Medicine, University of Nottingham,

Nottingham, UK

e-mail: kl.cheung@nottingham.ac.uk

H. M. Holmes

Division of Geriatric and Palliative Medicine,

University of Texas Health Science Center

McGovern Medical School, Houston, USA
Keywords: Cancer trials; Clinical trials; Geriatric assessment; Older adults

\section{Key Summary Points}

Older adults remain under-represented in cancer clinical trials, despite a willingness to participate in research.

Alternative study designs may be considered to address the fact that older adults potentially have different treatment goals compared to the younger population.

When securing trial funding, special consideration should be made to fund additional resources specific to older adults including integration of geriatric assessment and assistance with transport and communication.

Patients with cognitive impairment should not be automatically excluded from inclusion in trials.

Trial sponsors have a duty to raise awareness and include older adults as appropriate. 


\section{DIGITAL FEATURES}

This article is published with digital features, including a summary slide, to facilitate understanding of the article. To view digital features for this article go to https://doi.org/10.6084/ m9.figshare.13547645.

\section{INTRODUCTION}

Our population is ageing; virtually every country in the world is experiencing growth in the number and proportion of older adults [1]. An ageing population increases the requirements for health care systems, social services support and changing family dynamics.

In 2017, two out of six deaths in the world were from cancer, the second cause of death after cardiovascular disease. Of these deaths, almost half $(46 \%)$ were in patients over 70 years of age [2]. This is likely a consequence of three main factors: (1) as the population increases, so does the annual number of deaths, (2) the world is getting wealthier and fewer people die prematurely [3], and (3) cancer is a disease of ageing: as the global population ages, the incidence and prevalence of cancer increases.

There is a lack of representation of older adults and other vulnerable populations in clinical trials across all areas of medicine [4], including in the oncology setting $[5,6]$; only around $25 \%$ of cancer trials have enrolled participants aged $\geq 65$ years [7]. This has huge implications for treatment of older adults with cancer. Firstly, there is a lack of good clinical evidence in the older age group for health care professionals and patients to make informed treatment decisions. Medical bodies such as the National Institutes for Health in the USA and the National Institute for Health and Care Excellence in the UK base treatment guidelines and recommendations on trials primarily of younger adults with cancer. As a result, the medical community is providing cancer care to older adults based on evidence that was acquired in a different population: evidence typically from patients with fewer comorbidities, less polypharmacy, better overall health and different physiology [8].
A summary of the challenges discussed in this article and potential solutions is given in Table 1.

\section{CHALLENGES}

Reasons for the lack of inclusion in trials are multifactorial and challenging. The main challenges in recruiting older adults to clinical trials can be divided into three areas, which contain some overlapping themes: (1) protocol factors including recruitment and study design, (2) patient factors including motivation and competing comorbidities, and (3) sponsor factors including motives of sponsors and relevant funding agencies.

\section{Protocol Factors}

The literature suggests that older adults do want to be involved in clinical trials [9] and find inclusion a positive experience, even in studies with a negative or neutral outcome [10]. However, they do not actively seek out trials they can participate in [11]. Therefore, strategies for recruitment to studies and trial design should be specifically tailored to older adults.

\section{Recruitment}

The number of older patients who need to be approached to recruit significant numbers to a trial is much greater than their younger counterparts [12]. In a review of 14 published randomised controlled trials in various specialties in older adults by McMurdo et al. [12], the authors found that the number needed to screen to recruit one older participant was three, due to patient refusal to participate, for various reasons, increasing the burden of work on trialists.

There is inherent bias on the part of the clinician and recruiting health care team in the selection of patients for a clinical trial. A retrospective case-controlled study of patients with breast cancer by Kemeny et al. [13] sought to determine whether older patients were as likely as younger patients to be offered and to accept treatment in a clinical trial. The study 
Table 1 A summary of the challenges facing recruitment of older adults to cancer clinical trials and potential solutions to overcome them

\begin{tabular}{|c|c|c|}
\hline & Challenges & Potential solutions \\
\hline $\begin{array}{l}\text { Protocol } \\
\text { factors }\end{array}$ & $\begin{array}{l}\text { Recruitment } \\
\text { Older adults do want to be included in trials, but are } \\
\text { less likely to be asked to participate } \\
\text { Different forms of communication and advertisement } \\
\text { may be required } \\
\text { Study design } \\
\text { In non-blinded trials, clinician bias towards a particular } \\
\text { treatment arm may result in non-inclusion of specific } \\
\text { patients } \\
\text { Older patients may have a pre-existing preference to } \\
\text { undergo or avoid certain therapies, even if survival is } \\
\text { impacted }\end{array}$ & $\begin{array}{l}\text { Recruitment } \\
\text { Older adults may require additional support, and } \\
\text { appropriate time and resources should be allocated } \\
\text { here } \\
\text { Study design } \\
\text { Alternative study designs such as prospective cohort } \\
\text { studies or retrospective evaluations should be } \\
\text { considered } \\
\text { Endpoints of a trial should be modified to meet the } \\
\text { needs of older adults }\end{array}$ \\
\hline $\begin{array}{l}\text { Patient } \\
\text { factors }\end{array}$ & $\begin{array}{l}\text { Motivation } \\
\text { Maintaining quality of life may be more important } \\
\text { than prolonging survival } \\
\text { Positive relationships between study staff and } \\
\text { participants are important } \\
\text { Competing comorbidity } \\
\text { Physical obstacles which impede participation in a trial } \\
\text { include mobility issues, communication difficulty, } \\
\text { economic constraints and frailty } \\
\text { Patients with cognitive impairment are usually } \\
\text { excluded from clinical trials }\end{array}$ & $\begin{array}{l}\text { Motivation } \\
\text { Trial funding should include consideration of } \\
\text { transportation of participants, additional } \\
\text { communication needs and supportive services } \\
\text { Competing comorbidity } \\
\text { Integration of geriatric assessment as routine in } \\
\text { cancer clinical trials } \\
\text { Standard protocols for inclusion of older adults with } \\
\text { cognitive impairment with support from carers and } \\
\text { family }\end{array}$ \\
\hline $\begin{array}{l}\text { Sponsor } \\
\text { factors }\end{array}$ & $\begin{array}{l}\text { There is under-representation of older adults in trials } \\
\text { by regulatory bodies } \\
\text { Inappropriate age limits and exclusion of frail patients } \\
\text { No penalty to sponsors for not including older adults }\end{array}$ & $\begin{array}{l}\text { Ban on upper age limit for inclusion in clinical trials } \\
\text { Penalty for not providing information on number of } \\
\text { older adults recruited } \\
\text { Pressure on sponsors to raise awareness of these issues }\end{array}$ \\
\hline
\end{tabular}

demonstrated that older patients were significantly less likely to be offered a clinical trial (35\% versus $51 \%, p=0.006)$, however, if offered, they were as likely as their younger counterparts to accept enrolment. This suggests that unconscious ageism is present within the health care team.
An anonymous survey of approximately 1000 members of the Alliance for Clinical Trials in Oncology group surveyed the opinion of health care professionals, patient advocates and research staff. Overall, one third felt that $>50 \%$ of clinical trials enrolees should be aged 65 or above, and $64.8 \%$ felt that there was 
improvement to be made in the enrolment of older patients [14].

Methods of recruitment to clinical trials may need to be tailored to older adults to overcome barriers including communication deficits, negative perceptions of potential trial participants and additional concerns from older adults [15]. Harris et al. [16] compared methods of recruitment by telephone or questionnaire to a physical activity study in 560 older adults. Telephone contact increased recruitment to the study by $10 \%$ (95\% CI $0.2-19.8 \%)$, but inclusion of a questionnaire did not. Telephone contact may have given the potential study participants greater time and opportunity to speak to a research nurse and receive further information about the study if required. This should be considered when designing a study; more time and resources may need to be allocated to provide this service.

\section{Study Design}

Increasing access for older adults to trials may be hindered by trial design. For example, randomised controlled trials of treatment versus non-treatment or comparing two different treatments, where the treatment(s) has potential side effects, with little patient benefit, such as chemotherapy, may be less attractive to older adults where maintenance of quality of life may outweigh curative intent [17].

Using breast cancer as an example, there are a number of notable trials in older women that have failed to recruit significant numbers for reasons related to both trial design and patient factors. The Endocrine \pm Surgical Therapy for Elderly women with Mammary cancer (ESTEeM) trial randomised women $\geq 75$ years of age with invasive, operable, moderate or strongly oestrogen receptor (ER)-positive breast cancer to receive primary endocrine therapy (PET) or surgery and adjuvant endocrine therapy for 5 years [18]. The trial aimed to recruit 1200 patients; however, recruitment was extremely slow, and the study only managed to randomise nine patients and therefore closed early.

The Adjuvant Cytotoxic Chemotherapy in Older women (ACTION) trial randomised women $\geq 70$ years of age with primary breast cancer treated by surgery, who were defined as high-risk of recurrence and had tumours which were ER-negative or weakly positive, to receive adjuvant chemotherapy or no further treatment [19]. The initial phase of the trial aimed to recruit 200 patients to evaluate the accrual rate and tolerance of chemotherapy. Unfortunately, recruitment was low and the trial was closed early.

Although both studies address important questions in this patient population, they were difficult to conduct as randomised trials due to the major differences in treatment arms, i.e. surgery or PET in the ESTEeM trial and chemotherapy or no chemotherapy in the ACTION trial, as it was not possible to blind the treatment options. This can cause bias in that the clinician may have a preference towards one treatment arm for a particular patient. Furthermore, invited patients may have had a pre-existing preference to undergo or avoid certain therapies from the outset [20] and may be averse to a particular treatment, even if it could improve their survival [21].

Therefore, it could be suggested that randomised controlled trials are not the preferable method of choice to answer the hypothesis of these studies in the older population. Alternative options, such as prospective cohort studies or retrospective evaluation of national population-based data sets, may answer questions regarding oncological outcomes based on what treatment the patient received [22].

\section{Patient Factors}

There are a number of patient-related factors to consider for older adults who are approached to be included in a clinical trial, including patient motivation for inclusion and treatment goals, as well as competing comorbidities, frailty and how this might impact on physical participation.

\section{Motivation}

As demonstrated by the ESTEeM and ACTION trials, older adults may be less inclined to participate in clinical trials which have a treatment 
arm with potential side effects which may impact quality of life.

Moorcraft et al. [23] invited all patients who had been approached about participation in clinical trials at the gastrointestinal and lymphoma unit at their centre to complete a questionnaire about their experience. A total of $88 \%$ of 276 patients were approached and consented to participate in a clinical trial. Interestingly, increased age ( $\geq 65$ years) was not a factor as to whether or not they would participate in a trial $(p=0.236)$, nor was performance status $(p=0.839)$. The most important reason for trial participation irrespective of age was a belief that 'the trial offered the best treatment available' or that 'the trial results could benefit others'. Interestingly, patients were more likely to state that their main reason for participation was 'the trial results could benefit others' if they were $<65$ years of age compared to $\geq 65$ years (64\% versus 39\%). This is important to consider when approaching an older adult to participate in a trial; maintenance of their own quality of life may be the most important factor to them when considering treatment.

When investigating why older adults participated in a randomised trial of vascular disease prevention, Tolmie et al. [24] found that curiosity, self-interest and altruism were important motivators. Study subjects were likely to continue in the trial if positive relationships were formed between study staff and participants, and if participants were kept informed of the progress of the study at regular intervals. This is an important point to note in cancer trials, which may involve treatments with more severe side effects, such as chemotherapy.

Although there is limited evidence to establish why older adults participate in clinical trials, particularly trials related to cancer, the takehome message is that older adults do participate in clinical trials when asked, although their motives for doing so may be different compared to their younger counterparts.

\section{Competing Comorbidities}

Greater likelihood of comorbidity in older adults increases concerns of side effects from additional treatments as well as placing physical and psychological burdens on an older adult. Typically, older people face a combination of obstacles including comorbidity, economic constraints, communication issues and physical immobility that constrains transportation options [7, 25]. They may have additional accessibility needs such as help with reading study material due to deterioration in eyesight or help with understanding study instructions due to hearing loss.

A huge issue arises concerning older patients with cognitive impairment being automatically excluded from clinical trials due to issues regarding consent. This is particularly concerning, as fewer patients with dementia present with cancer at an early stage and are less likely to have surgery versus non-surgical treatment options [26]. There is an increasing global burden of dementia; 40-50 million people are currently living with dementia [27], and this is predicted to double every 20 years [28]. Therefore, cognitive impairment in a patient should be treated as any other comorbidity affecting the older population [29], and adjustments should be made to allow these patients to participate in clinical trials. Specifically when considering patients with a diagnosis of dementia, the stage of their disease journey is important. Dementia is a disease with a very long course; patients with milder disease earlier in the course are still likely to retain decisionmaking ability and should be offered trial inclusion.

\section{Sponsor Factors}

Under-representation of older adults in trials by regulatory agencies such as the National Institute for Health Research in the UK and the Food and Drug Administration (FDA) in the USA has been shown [30]. Conscious ageism occurs in study design with inappropriate age limits and exclusion of frail older patients [25]. Bayer and Tadd [31] examined studies submitted to a local research ethics committee and found that 20 out of 46 approved studies had an upper age limit that seemed unjustifiable.

Lewis et al. [32] retrospectively analysed clinical trials sponsored by the National Cancer 
Institute (NCI) in the USA, accounting for over 59,000 patients from 1997 to 2000 . They found that only $32 \%$ of participants were older adults, compared with $61 \%$ of patients with incident cancers. More recent data from the NCI (period 2001-2011) did not show any improvement in this area [33].

In addition, Hernandez-Torres et al. [34] looked at whether accrual of older adults to trials led by the Canadian Cancer Trials Group had increased from 2003 to the present time and whether exclusion criteria had broadened. Although there was a small increase in inclusion, older adults remained under-represented.

Sponsors and regulatory agencies may be more interested in the younger population, as publicity from successful results is often more pronounced in this cohort. Younger participants are less likely to have comorbidities that could place them at higher risk for adverse outcomes. Currently, there is no penalty for exclusion and therefore no incentive for sponsors to promote the inclusion of older adults.

\section{POTENTIAL SOLUTIONS}

Many organisations including the American Society of Clinical Oncology, the International Society of Geriatric Oncology (SIOG) and the European Organisation for Research and Treatment of Cancer have issued recommendations on strengthening the evidence base for treating older patients with cancer by increasing their enrolment in clinical trials and improving trial design [35].

\section{Protocol Factors}

\section{Recruitment}

It is accepted by older adults and health care professionals that different methods of recruitment to clinical trials is required to engage older adults [12]; however, the type of additional support and how this should be delivered remains uncertain.

Greater support for older patients, for example, follow-up telephone consultations to check for understanding of patient information sheets, has been found to be helpful [36], as well as additional research staff available in clinics to explain potential trials to patients and provide educational materials to the patient and health care team regarding potential treatment toxicity [37]. However, other methods, such as receipt of educational materials and financial incentives, have not been found to be helpful. Kimmick et al. [38] conducted a randomised trial comparing educational intervention with standard information to improve accrual of cancer patients aged $\geq 65$ years to group-sponsored treatment trials. Educational intervention consisted of an educational seminar, educational materials, email reminder and a case discussion seminar. Accrual of older patients was not increased by this particular intervention.

The Medicare programme in the USA conducted a longitudinal analysis of the enrolment of older patients in NCI-sponsored cancer trials from 1996-2003 [39]. The study compared recruitment of older women to trials before and after a reimbursement policy change was introduced. The policy change extended reimbursement to cover participants of clinical trials. The study found that a change in reimbursement policy was not associated with increased enrolment of older patients in cancer trials.

Flexibility in the method of recruitment is paramount, and investigators should not be discouraged by a seeming lack of participation; alternative methods should be planned from the outset.

\section{Study Design}

The traditional design of randomised controlled trials may not be the most appropriate design for older adults, and there are a number of potential alternative designs to consider [40]. Potential alternatives include prospective cohort studies, the use of national populationbased data sets, or randomised trials with loose inclusion/exclusion criteria, including pragmatic trials. In response to the lack of success in randomised controlled trials assessing adjuvant chemotherapy in older women with breast cancer, alternative study designs have been sought.

Gray et al. [41] conducted a retrospective cohort study in patients aged $\geq 70$ years with 
early breast cancer, with and without high-level comorbidity, identified from the Scottish Cancer Registry, with data linked to other health records. They were able to identify 9653 patients with low comorbidity and 7965 with high comorbidity. Propensity score matching was used to estimate the effect of chemotherapy on breast cancer mortality and overall survival, adjusting for differences in prognosis between those who received chemotherapy and those who did not. The average predicted benefit of chemotherapy was an additional three out of every 100 women surviving for 10 years, and an additional four out of every 100 for those with comorbidity. The authors concluded that the relative effectiveness of adjuvant chemotherapy in older women appears similar to that in younger women (recruited to clinical trials), and this would imply that estimates of treatment effectiveness among trial-eligible patients are generalisable to trial-under-represented groups.

A prospective cohort study was the approach taken by Fietz et al. [42] in Germany. The team recruited 2316 breast cancer patients, including $478 \geq 70$ years, to a prospective registry which included detailed patient and tumour characteristics, treatment details and oncological outcome. The proportion of patients receiving taxane-based adjuvant chemotherapy was similar independent of age (61\% in the younger cohort versus $62 \%$ in the older cohort). However, older patients with hormone receptor positive tumours were more likely to receive adjuvant endocrine therapy alone. Amongst the patients who did receive adjuvant chemotherapy, disease free survival was comparable between younger and older patients $(86 \%$ versus $88 \%$ at 3 years).

Randomised controlled trials with some element of blinding may still be possible in older adults, for example single-blinding of the interventionist to allocation or control arm, where older participants are not blinded to the treatment, may encourage their participation [43].

Endpoints of a trial should potentially be modified in older adults. For example, instead of overall survival or progression-free survival, investigators might consider quality of life, toxicity of treatment and maintaining functional independence as markers of treatment outcome in older adults. Measurement of disease-specific survival is useful as it indicates the number of patients who actually die as a result of cancer as opposed to other chronic conditions [44].

Involvement of older adults through patient and public involvement schemes, in order to identify the most appropriate trial design, is paramount.

\section{Decentralised Clinical Trials}

In recent times, there has been a dramatic increase in the use of technology to help reduce the burden placed on participants in clinical trials. The urgent need for increased technology know-how and user ability has been dramatically highlighted by the current coronavirus pandemic.

The term 'decentralised clinical trials' is used to define those executed through telemedicine and mobile or local health care providers, using processes and technologies that differ from the traditional clinical trial model [45]. Examples include the use of telemedicine, teleconsent, wearable devices and smartphone applications for educational material, reminders and collection of patient-reported outcomes [46].

In order to ensure fluidity of the process, there are a number of issues of basic technology that must be overcome, including enabling a secure and reliable telephone/video contact, as well as financial reimbursement and legal and regulatory issues [46].

Some examples specific to older adults in cancer clinical trials include the use of electronic signature to avoid unnecessary hospital visits for consenting, virtual screening assessment to determine eligibility for investigation and treatment, and routine follow-up appointments [47].

The rapid expansion and understanding of how technology can be integrated into health services in 2020 is exciting and, in addition to current face-to-face services, can only improve access for older adults to clinical trials. 


\section{Patient Factors}

\section{Motivation}

Older patients do not view age as an important reason for refusing clinical trials [48]. Therefore, we must consider how we can make participation in clinical trials easier for them. Trial information should be provided in age-appropriate formats, which may include large-print information leaflets and additional audio-visual material for the hearing- and vision-impaired [7]. When applying for trial funding, funds should be considered to facilitate access to supportive services for older adults, for example, a research nurse trained in geriatrics, additional funding for transportation, and access to information regarding social services and support.

\section{Competing Comorbidity}

The FDA guidance 'Cancer Clinical Trial Eligibility Criteria: Patients with Organ Dysfunction or Prior or Concurrent Malignancies', published in July 2020, clearly states that comorbidity should not be a reason to automatically exclude a person from a clinical trial, and provides practical advice related to types of comorbidity including renal, cardiac and hepatic problems [49].

This can be achieved by inclusion of geriatric assessment (GA) in clinical trials and robust guidance for inclusion of patients with cognitive impairment.

Geriatric assessment is recommended by SIOG to be integrated into geriatric oncology, but how it should be implemented and the results acted upon remains less clear [50]. In a clinical trial setting, there are two potential uses for GA. Firstly, trials which involve different treatment allocations should embed some form of GA into their design so that patients can be allocated to a particular trial arm or treated specifically according to their needs as identified by GA, rather than simply being excluded from a trial because they are too frail. Secondly, all trials involving older adults should include some form of GA in their protocol, to identify specific needs of this population that can be addressed to improve their overall quality of life, general health and potentially the ability to continue participating in the trial.

An example of a randomised trial designed specifically for older patients that used geriatric assessment tools is the Cancer and Leukemia Group B (CALGB) 49907 trial [51], which compared two different types of adjuvant chemotherapy in older women with breast cancer and nested within the trial a detailed quality-of-life study [52]. Geriatric assessment tools were integrated into the study to provide insights on the quality of life of study participants while also addressing issues detected.

There are some geriatric assessment-based tools, such as the Cancer and Ageing Research Group (CARG) chemotherapy risk score [53] and the Chemotherapy Risk Assessment Scale for High-Age Patients (CRASH) [54], that have been shown to be superior to standard measures for predicting chemotherapy toxicity in older adults. These tools would be useful additions to any clinical trial protocol involving prescription of chemotherapy.

Inclusion of older adults with dementia requires support from a patient's family and care network, health care professionals, and societies and organisations involved in the care of older adults with dementia.

A number of resources have been designed to help include older adults with dementia in clinical trials. The National Institute on Aging Imbedded Pragmatic Alzheimer's Disease (AD) and AD-Related Dementias Clinical Trials (IMPACT) Collaboratory has convened a Design and Statistics Core [55], the goals of which are to support the design and conduct of embedded pragmatic clinical trials in older adults with dementia. The roles of this body include education of scientists and health care providers in trial design, production of literature to address relevant challenges, designing potential interventions to tackle the challenges of multiple comorbidities, and the incorporation of multiple health care systems and stakeholders [56].

The Alzheimer's Association has developed a three-item questionnaire that can be integrated into the informed consent process to assess whether patients with Alzheimer's disease do have the capacity to make informed decisions regarding participation in treatment [57]. This 
tool is designed to be quick and simple to administer and could be incorporated into routine clinical practice and trial recruitment.

Some studies have included proxy consent as acceptable in their inclusion criteria. In the UK Bridging the Age Gap in Breast Cancer study of 3375 older women, patients without cognitive capacity were eligible to participate if a relative or friend was willing to sign proxy consent [58].

With increased awareness of the importance of recruiting older adults with cognitive impairment and potential solutions, health care professionals and scientists are more likely to include defined protocols in their study proposals.

\section{Sponsor Factors}

The FDA have released guidance regarding the inclusion of older adults in trials. Their Guidance for Industry $[59,60]$ encourages the fair representation of older adults in clinical trials, emphasising the importance of considering common conditions related to ageing and guidelines for geriatric labelling on drugs. Although the guidelines do not require manufacturers to include sufficient numbers of older adults in clinical trials, they encourage information on the package insert regarding the number of older adults recruited, thus raising awareness of the issue $[59,60]$. The FDA have more recently made renewed efforts to promote inclusion of older adults in clinical trials, by promoting the establishment of partnerships to provide travel assistance, updated guidance on payment and reimbursement of research participants [61], and discouraging upper age limits for trials [62].

Further efforts should be made towards improvements in this area, including a ban on upper age limits for inclusion in clinical trials and standardised protocols for sponsors to use to allow routine inclusion of patients with cognitive impairment. The American Society of Clinical Oncology are in support of overarching bodies to exert authority in this way; they have developed recommendations which include leverage on research designs and infrastructure for trials involving older adults and increasing the authority of the FDA to incentivise and require research involving older adults with cancer [30].

\section{CONCLUSIONS}

This article has outlined the challenges and solutions to consider in the recruitment of older adults to cancer clinical trials. Health care providers should be aware that a variety of methods may be required to recruit greater numbers of older adults, including additional staff and time spent on going through trial details, follow-up telephone calls and accessible information. When designing studies for older adults with cancer, trialists should be flexible in inclusion criteria, study design and endpoints. Patients with cognitive impairment should not be excluded from participation in trials, and consent from family or carers should be considered as routine. Geriatric assessment in some form should be embedded as standard in clinical trials of older adults with cancer. There needs to be increased pressure on sponsors to make the inclusion of older adults in clinical trials mandatory and to make the numbers recruited available in the public domain. There is an urgent need to address these issues on a global scale. Moving forward, one option is for a geriatrician to work alongside an oncologist in the context of research for older adults, similar to what is being implemented in clinical service delivery. Most importantly, older patients and their carers should be considered key stakeholders in clinical trials and actively involved in all stages of the trial process from inception.

\section{ACKNOWLEDGMENTS}

Funding. No funding or sponsorship was received for publication of this article. This review was conducted as part of RM Parks' PhD, supported by a fellowship funded by Nottingham Hospitals Charity, UK, and an honorary fellowship from the Royal College of Surgeons of England, UK. HM Holmes is funded by Blue 
Cross/Blue Shield for a research grant unrelated to this manuscript.

Authorship. All named authors meet the International Committee of Medical Journal Editors (ICMJE) criteria for authorship for this article, take responsibility for the integrity of the work as a whole, and have given their approval for this version to be published.

Disclosures. Kwok-Leung Cheung is a member of Oncology and Therapy's Editorial board but has nothing else to disclose. Ruth M. Parks and Holly M. Holmes have nothing to disclose.

Compliance with Ethics Guidelines. This article is based on previously conducted studies and does not contain any studies with human participants or animals performed by any of the authors.

Data Availability. Data sharing is not application to this article as no data sets were generated or analysed during the current study.

Open Access. This article is licensed under a Creative Commons Attribution-NonCommercial 4.0 International License, which permits any non-commercial use, sharing, adaptation, distribution and reproduction in any medium or format, as long as you give appropriate credit to the original author(s) and the source, provide a link to the Creative Commons licence, and indicate if changes were made. The images or other third party material in this article are included in the article's Creative Commons licence, unless indicated otherwise in a credit line to the material. If material is not included in the article's Creative Commons licence and your intended use is not permitted by statutory regulation or exceeds the permitted use, you will need to obtain permission directly from the copyright holder. To view a copy of this licence, visit http://creativecommons.org/licenses/bync/4.0/.

\section{REFERENCES}

1. United Nations. Ageing. 2019. https://www.un.org/ en/sections/issues-depth/ageing/. Accessed 21 Oct 2020.

2. Our World in Data. University of Oxfrod GCDL. 2020. https://ourworldindata.org. Accessed 24 Sep 2020.

3. Fonseca V, Caeiro J. Bioethics and healthcare policies. The benefit of using genetic tests of BRCA 1 and BRCA 2 in elderly patients. Int J Health Plann Manag. 2020. https://doi.org/10.1002/hpm.3072.

4. Mody L, Miller DK, McGloin JM, Freeman M, Marcantonio ER, Magaziner J, et al. Recruitment and retention of older adults in aging research. J Am Geriatr Soc. 2008;56(12):2340-8.

5. Murthy VH, Krumholz HM, Gross CP. Participation in cancer clinical trials: race-, sex-, and age-based disparities. JAMA. 2004;291(22):2720-6.

6. Institute of Medicine Committee on Cancer Research Among M, The Medically U. The National Academies Collection: Reports funded by National Institutes of Health. In: Haynes MA, Smedley BD, editors. The unequal burden of cancer: an assessment of NIH research and programs for ethnic minorities and the medically underserved. Washington: National Academies Press; 1999.

7. Herrera AP, Snipes SA, King DW, Torres-Vigil I, Goldberg DS, Weinberg AD. Disparate inclusion of older adults in clinical trials: priorities and opportunities for policy and practice change. Am J Public Health. 2010;100(Suppl 1):S105-12.

8. Outlaw D, Williams GR. Is the lack of evidence in older adults with cancer compromising safety? Expert Opin Drug Saf. 2020;19(9):1059-61.

9. van der Cammen TJM, Crome P. Persistent exclusion of older people from clinical trials of cardiovascular and antithrombotic medicinal products. Eur Geriatr Med. 2018;9(4):413-4.

10. Yuval R, Uziel K, Gordon N, Merdler A, Khader N, Karkabi B, et al. Perceived benefit after participating in positive or negative/neutral heart failure trials: the patients' perspective. Eur J Heart Fail. 2001;3(2): 217-23.

11. Townsley CA, Chan KK, Pond GR, Marquez C, Siu LL, Straus SE. Understanding the attitudes of the elderly towards enrolment into cancer clinical trials. BMC Cancer. 2006;6:34.

12. McMurdo MET, Roberts H, Parker S, Wyatt N, May $\mathrm{H}$, Goodman $\mathrm{C}$, et al. Improving recruitment of 
older people to research through good practice. Age Ageing. 2011;40(6):659-65.

13. Kemeny MM, Peterson BL, Kornblith AB, Muss HB, Wheeler J, Levine E, et al. Barriers to clinical trial participation by older women with breast cancer. J Clin Oncol. 2003;21(12):2268-75.

14. Freedman RA, Dockter TJ, Lafky JM, Hurria A, Muss HJ, Cohen HJ, et al. Promoting accrual of older patients with cancer to clinical trials: an Alliance for Clinical Trials in Oncology member survey (A171602). Oncologist. 2018;23(9):1016-23.

15. McHenry JC, Insel KC, Einstein GO, Vidrine AN, Koerner KM, Morrow DG. Recruitment of older adults: success may be in the details. Gerontologist. 2012;55(5):845-53.

16. Harris TJ, Carey IM, Victor CR, Adams R, Cook DG. Optimising recruitment into a study of physical activity in older people: a randomised controlled trial of different approaches. Age Ageing. 2008;37(6):659-65.

17. Estapé T. Cancer in the elderly: challenges and barriers. Asia Pac J Oncol Nurs. 2018;5(1):40-2.

18. Cancer Research UK. A trial looking at anastrozole with or without surgery for older women with breast cancer (ESTEeM). 2011. https://www. cancerresearchuk.org/about-cancer/find-a-clinicaltrial/a-trial-looking-at-anastrozole-with-or-withoutsurgery-for-older-women-with-breastcancer\#undefined. Accessed 8 Oct 2020.

19. Cancer Research UK. A trial comparing adjuvant chemotherapy with standard treatment for older women with early stage breast cancer (ACTION). 2010. https://www.cancerresearchuk.org/aboutcancer/find-a-clinical-trial/a-trial-comparingadjuvant-chemotherapy-with-standard-treatmentfor-older-women-with-early-stage-breast-cancer. Accessed 8 Oct 2020.

20. Ring A, Reed M, Leonard R, Kunkler I, Muss H, Wildiers $\mathrm{H}$, et al. The treatment of early breast cancer in women over the age of 70 . Br J Cancer. 2011;105(2):189-93.

21. Reed MWR, Wyld L, Ellis P, Bliss J, Leonard R. Breast cancer in older women: trials and tribulations. Clin Oncol. 2009;21(2):99-102.

22. Leonard R, Ballinger R, Cameron D, Ellis P, Fallowfield L, Gosney M, et al. Adjuvant chemotherapy in older women (ACTION) study: what did we learn from the pilot phase? $\mathrm{Br} \mathrm{J}$ Cancer. 2011;105(9):1260-6.

23. Moorcraft SY, Marriott C, Peckitt C, Cunningham D, Chau I, Starling N, et al. Patients' willingness to participate in clinical trials and their views on aspects of cancer research: results of a prospective patient survey. Trials. 2016;17:17.

24. Tolmie EP, Mungall MMB, Louden G, Lindsay GM, Gaw A. Understanding why older people participate in clinical trials: the experience of the Scottish PROSPER participants. Age Ageing. 2004;33(4): 374-8.

25. Lindley RI. Drug trials for older people. J Gerontol. 2011;67A(2):152-7.

26. Raji MA, Kuo Y-F, Freeman JL, Goodwin JS. Effect of a dementia diagnosis on survival of older patients after a diagnosis of breast, colon, or prostate cancer: implications for cancer care. Arch Intern Med. 2008;168(18):2033-40.

27. Collaborators GBDD. Global, regional, and national burden of Alzheimer's disease and other dementias, 1990-2016: a systematic analysis for the Global Burden of Disease Study 2016. Lancet Neurol. 2019;18(1):88-106.

28. van der Willik KD, Schagen SB, Ikram MA. Cancer and dementia: two sides of the same coin? Eur J Clin Investig. 2018;48(11):e13019e.

29. Mary Parks R, Jauhari Y, Morgan J, Wyld L, Cheung K-L. Special issues for older women with primary breast cancer. Breast Cancer Management. 2020;9(3):BMT43.

30. Hurria A, Levit LA, Dale W, Mohile SG, Muss HB, Fehrenbacher L, et al. Improving the evidence base for treating older adults with cancer: American Society of Clinical Oncology Statement. J Clin Oncol. 2015;33(32):3826-33.

31. Bayer A, Tadd W. Unjustified exclusion of elderly people from studies submitted to research ethics committee for approval: descriptive study. BMJ. 2000;321(7267):992-3.

32. Lewis JH, Kilgore ML, Goldman DP, Trimble EL, Kaplan R, Montello MJ, et al. Participation of patients 65 years of age or older in cancer clinical trials. J Clin Oncol. 2003;21(7):1383-9.

33. Hurria A, Dale W, Mooney M, Rowland JH, Ballman $\mathrm{KV}$, Cohen HJ, et al. Designing therapeutic clinical trials for older and frail adults with cancer: U13 conference recommendations. J Clin Oncol. 2014;32(24):2587-94.

34. Hernandez-Torres C, Cheung WY, Kong S, O'Callaghan CJ, Hsu T. Accrual of older adults to cancer clinical trials led by the Canadian cancer trials group. Is trial design a barrier? J Geriatr Oncol. 2020;11(3):455-62. 
35. Soto-Perez-De-Celis E, Lichtman SM. Considerations for clinical trial design in older adults with cancer. Expert Opin Investig Drugs. 2017;26(10): 1099-102.

36. Chatters R, Newbould L, Sprange K, Hind D, Mountain G, Shortland K, et al. Recruitment of older adults to three preventative lifestyle improvement studies. Trials. 2018;19(1):121.

37. Hurria A. Cancer Network. Clinical Trials in Older Adults with Cancer: past and future. 2007. https:// www.cancernetwork.com/view/clinical-trials-olderadults-cancer-past-and-future. Accessed 14 Oct 2020.

38. Kimmick GG, Peterson BL, Kornblith AB, Mandelblatt J, Johnson JL, Wheeler J, et al. Improving accrual of older persons to cancer treatment trials: a randomized trial comparing an educational intervention with standard information: CALGB 360001. J Clin Oncol. 2005;23(10):2201-7.

39. Gross CP, Wong N, Dubin JA, Mayne ST, Krumholz HM. Enrollment of older persons in cancer trials after the medicare reimbursement policy change. Arch Intern Med. 2005;165(13):1514-20.

40. Clegg A, Relton C, Young J, Witham M. Improving recruitment of older people to clinical trials: use of the cohort multiple randomised controlled trial design. Age Ageing. 2015;44(4):547-50.

41. Gray E, Marti J, Wyatt JC, Brewster DH, Hall PS, Group SA. Chemotherapy effectiveness in trial-underrepresented groups with early breast cancer: a retrospective cohort study. PLoS Med. 2019;16(12): e1003006.

42. Fietz T, Zahn M-O, Köhler A, Engel E, Frank M, Kruggel L, et al. Routine treatment and outcome of breast cancer in younger versus elderly patients: results from the SENORA project of the prospective German TMK cohort study. Breast Cancer Res Treat. 2018;167(2):567-78.

43. Allore HG, Tinettia ME, Gill TM, Peduzzi PN. Experimental designs for multicomponent interventions among persons with multifactorial geriatric syndromes. Clinical Trials. 2005;2(1):13-21.

44. Whelehan S, Lynch O, Treacy N, Gleeson C, Oates A, O'Donovan A. Optimising clinical trial design in older cancer patients. Geriatrics (Basel, Switzerland). 2018;3(3):34.

45. Clinical Trials Transformation Initiative (CTTI). CTTI Recommendations: decentralized Clinical Trials. 2018. https://www.ctti-clinicaltrials.org/ projects/decentralized-clinical-trials. Accessed 18 Jan 2020.
46. Saini KS, de Las HB, Plummer R, Moreno V, Romano $\mathrm{M}$, de Castro J, et al. Reimagining global oncology clinical trials for the postpandemic era: a call to arms. JCO Glob Oncol. 2020;6:1357-62.

47. Doherty GJ, Goksu M, de Paula BHR. Rethinking cancer clinical trials for COVID-19 and beyond. Nat Cancer. 2020;1:568-72.

48. Townsley CA, Selby R, Siu LL. Systematic review of barriers to the recruitment of older patients with cancer onto clinical trials. J Clin Oncol. 2005;23(13):3112-24.

49. U.S. Department of Health and Human Services. Food and Drug Administration. Cancer Clinical Trial Eligibility Criteria: Patients with Organ Dysfunction or Prior or Concurrent Malignancies: Guidance for Industry. 2020. https://www.fda.gov/ media/123745/download. Accessed 18 Jan 2020.

50. Biganzoli L, Wildiers H, Oakman C, Marotti L, Loibl $S$, Kunkler I, et al. Management of elderly patients with breast cancer: updated recommendations of the International Society of Geriatric Oncology (SIOG) and European Society of Breast Cancer Specialists (EUSOMA). Lancet Oncol. 2012;13(4): e148-60.

51. Muss HB, Woolf S, Berry D, Cirrincione C, Weiss RB, Budman D, et al. Adjuvant chemotherapy in older and younger women with lymph node-positive breast cancer. JAMA. 2005;293(9):1073-81.

52. Cohen HJ, Muss HB. The Cancer and Leukemia Group B Cancer in the Elderly Committee: addressing a major cancer need. Clin Cancer Res. 2006;12(11):3606s

53. Hurria A, Mohile S, Gajra A, Klepin H, Muss H, Chapman A, et al. Validation of a Prediction Tool for Chemotherapy Toxicity in Older Adults With Cancer. J Clin Oncol. 2016;34(20):2366-71.

54. Extermann M, Boler I, Reich RR, Lyman GH, Brown $\mathrm{RH}$, DeFelice J, et al. Predicting the risk of chemotherapy toxicity in older patients: the Chemotherapy Risk Assessment Scale for High-Age Patients (CRASH) score. Cancer. 2012;118(13): 3377-86.

55. Mitchell SL, Mor V, Harrison J, McCarthy EP. Embedded pragmatic trials in dementia care: realizing the vision of the NIA IMPACT Collaboratory. J Am Geriatr Soc. 2020;68(Suppl 2):S1-s7.

56. Allore HG, Goldfeld KS, Gutman R, Li F, Monin JK, Taljaard $\mathrm{M}$, et al. Statistical considerations for embedded pragmatic clinical trials in people living with dementia. J Am Geriatr Soc. 2020;68(Suppl 2): S68-73. 
57. Palmer BW, Dunn LB, Appelbaum PS, Mudaliar S, Thal L, Henry R, et al. Assessment of capacity to consent to research among older persons with schizophrenia, Alzheimer disease, or diabetes mellitus: comparison of a 3-item questionnaire with a comprehensive standardized capacity instrument. Arch Gen Psychiatry. 2005;62(7):726-33.

58. Morgan JL, George J, Holmes G, Martin C, Reed MWR, Ward S, et al. Breast cancer surgery in older women: outcomes of the Bridging Age Gap in Breast Cancer study. Br J Surg. 2020;45:878.

59. US Food and Drug Administration Guidance for Industry. Content and format for geriatric labeling. 2001. https://www.fda.gov/media/72141/ download. Accessed 14 Oct 2020.
60. Food and Drug Administration. Inclusion of Older Adults in Cancer Clinical Trials: Guidance for Industry (draft guidance). 2020. https://www.fda. gov/media/135804/download. Accessed 18 Jan 2020.

61. US Food and Drug Administration. Payment and Reimbursement to Research Subjects. 2018. https:// www.fda.gov/regulatory-information/search-fdaguidance-documents/payment-andreimbursement-research-subjects. Accessed 14 Oct 2020.

62. US Food and Drug Administration. Good Review Practice: Clinical Review of Investigational New Drug Applications. 2013. https://www.fda.gov/ media/87621/download. Accessed 14 Oct 2020. 